\title{
Hypercapnic Ventilatory Response: A Comparison Between Elite and Novice Skin Divers
}

\author{
Dimitrios I. Bourdas, Theodoros S. Tsakiris, Athanasios I. Konstantopoulos, \\ Despoina V. Triantafillou and Nickos D. Geladas*
}

Department of Sports Medicine \& Biology of Exercise, Faculty of Physical Education and Sport Science, University of Athens, 17237 Daphne, Greece

\begin{abstract}
This study tested whether hypercapnic ventilatory response (HCVR) is affected by experience in apnoea and explored the possible underlying mechanisms of this phenomenon, with reference to maximum breath hold time (BHT).

Elite apnoea divers $(\mathrm{EBH}=11)$ and novice subjects $(\mathrm{NBH}=10)$ performed a HCVR test $(\mathrm{BM})$, which was repeated by the latter group on another day (PRE) after subjects executed five repeated maximum apnoeas. Subsequently, after a two week period of daily apnoea training, NBH subjects repeated HCVR test (POST). Diaphragmatic activity was recorded to determine apnoea easy going phase.

Baseline HCVR of EBH was not lower than that of NBH. After execution of five apnoeas, HCVR in EBH decreased $(\mathrm{P} \leq 0.05)$ whereas it was not different among BM, PRE, and POST conditions in the NBH. Higher BHT and easy going phase values ensued from apnoea maneuvers in EBH than in NBH (PRE and POST), and in NBH POST compared to PRE condition $(\mathrm{P} \leq 0.05)$. HCVR was highly correlated $(\mathrm{P} \leq 0.05)$ with the cumulative easy going phase in $\mathrm{EBH}$, $\mathrm{PRE}$ condition but nothing-similar observed in NBH, neither in PRE nor in POST conditions.

These results indicate that at rest HCVR is not lower in experienced, than novice, skin divers; this response becomes dull after five repeated maximum apnoeas only in EBH. In the NBH group, two weeks of apnoea training are not adequate to affect HCVR despite an increase of BHT. It appears that after a repeated maximum apnoeas maneuver experienced skin divers improve BHT by extending easy going phase whereas NBH by other means.
\end{abstract}

Keywords: Breath hold, $\mathrm{CO}_{2}$ sensitivity, easy going apnoea phase, repeated breath hold efforts.

\section{INTRODUCTION}

Breath responsiveness to a high concentration of carbon dioxide $\left(\mathrm{CO}_{2}\right)$ in arterial blood is referred as hypercapnic ventilatory response (HCVR). It is estimated by the slope $(\alpha)$ of the relation between ventilation ( $\left.\dot{\mathrm{V}}_{\mathrm{E}}\right)$ and partial pressure of end tidal $\mathrm{CO}_{2}(\alpha=\dot{\mathrm{V}} / \mathrm{PETCO} 2)$ and manifests the adaptability, health and welfare of pulmonary respiration [1].

Experienced skin divers because of familiarization and training seem to develop long term low chemosensitivity to $\mathrm{CO}_{2}$ [2-5], leading probably to a reduced need for ventilation and consequently in increased duration of breath hold time (BHT) [6]. Repeating breath hold efforts with a short time interval of $4 \mathrm{~min}$ between them, increases, temporarily though, $\mathrm{CO}_{2}$ retention [7]. Similarly, after five repeating breath hold efforts with face immersion in cold water at $12^{\circ} \mathrm{C}$ with an interval of $2 \mathrm{~min}$ between each effort, induce significant retention of $\mathrm{CO}_{2}$ which lasts at least $60 \mathrm{~min}$ in both experienced and novice divers [8]. It was speculated that repeated breath hold efforts through retention of $\mathrm{CO}_{2}$ resets in both novice and experienced divers, the sensitivity

*Address correspondence to this author at the Department of Sports Medicine \& Biology of Exercise, Faculty of Physical Education and Sport Science, University of Athens, 17237 Daphne, E. Antistassis 41, Greece;

Tel:+306977997102; E-mail: geladas@phed.uoa.gr threshold of central chemoreceptors [8] like apnoea training probably does and consequently both short apnoea repetition and training may reduce the need for breathing [9]. This phenomenon has been speculated to gradually lead in increased BHT in protocols of five repeated breath holds with an interval of 2-8 min [10-15]. However, all these assumptions were recently questioned since five repeated breath holds did not change HCVR in novice subjects and therefore recorded gradual BHT enhancement could not be attributed to HCVR alterations [16].

Collectively, dullness of hypercapnic ventilatory response is not observed indiscriminately in all skin divers and the influence of short and mid term training on it, remains entirely controversial. The scope of the present study was to explore the effect of short (five consecutive breath hold efforts) and mid term (two weeks of daily sessions) training on the development of low chemosensitivity in experienced and novice divers. It was assumed that hypercapnic ventilatory response is a trait, which befits to only experienced skin divers and short term training would not be effective in improving it.

\section{MATERIALS AND METHODS}

Subjects were grouped according to their experience in breath holding activities; the group of elite breath hold divers were members of apnoea national team $(\mathrm{EBH}, \mathrm{n}=11)$ with 
BHT greater than $3.5 \mathrm{~min}$ and the novice subjects were university students $(\mathrm{NBH}, \mathrm{n}=10)$ without any apnoea experience and BHT between $0.5 \mathrm{~min}$ and $3.5 \mathrm{~min}$. Their age, weight and height were $28.1 \pm 1.9$ years, $84.4 \pm 3.2 \mathrm{~kg}$, $182.5 \pm 1.8 \mathrm{~cm}$ and $20.9 \pm 0.5$ years, $82.2 \pm 3.6 \mathrm{~kg}, 180.2 \pm 2.1$ $\mathrm{cm}$ for $\mathrm{EBH}$ and NBH, respectively. Subjects in both groups had similar maximal oxygen uptake $\left(\dot{\mathrm{V}}_{2}\right.$ max $)$ ranging between 35 and $45 \mathrm{ml} / \mathrm{kg} / \mathrm{min}$.

\section{Experimental Procedures}

Initially, in separate days, at the same time, and in a random counter balanced order, subjects visited the laboratory twice. In the first visit, after familiarization and preliminary measurements, all subjects executed a HCVR test (BM condition). In particular, all participants familiarized themselves with the laboratory environment and the methods adopted in the study. They were instructed and trained in such a way so that each time an apnoea was performed, either in a laboratory or training setting, it was done in a relaxed way, in the same personal manner, without hyperventilating, swallowing or exhaling and performing any kind of maneuvers, like Valsava or Mueller [17]. Body measurements (Bilance SALUS S12, Italy), and a force vital capacity test in a sitting position (MedGraphics CPX/Ultima, USA) were also conducted. Subsequently, a HCVR test was performed, which was followed, within the next $30 \mathrm{~min}$, by a progressive effort till to exhaustion protocol, on a cycle ergometer (Lode ${ }^{\circledR}$ Instrumenten Holland), to measure $\dot{\mathrm{V}}_{2 \max }$ (MedGraphics CPX/Ultima, USA).

In the second visit (PRE condition) three days later, the subjects performed from a prone position, having the arms always beside their head at the same horizontal level, five repeated maximal-effort apnoeas $\left(\mathrm{BH}_{\mathrm{FI}}\right)$ with face immersion in cold water $\left(12^{\circ} \mathrm{C}\right)$ without being given any feedback of running time. An intermediate recovery period of 2 min was allowed among $\mathrm{BH}_{\mathrm{FI}}$. Briefly, under subjects' head was a water container (6.51) covered by a removable wooden pillow layered by a friendly soft hypoallergic material where the subjects rested their head and relaxed for 7 min until a stable resting heart rate was achieved. Subjects always wore a nose clip (PARADISIA, France) and breathed via a mouthpiece with two manual three way valves (Hans Rudolph. INC., K.C.MO., USA). The first valve was connected directly to automated open-circuit spirometer (MedGraphics CPX/Ultima, USA) in order to record respiratory variables. The second valve was connected to an elastic vinyl bag of 5 liters maximum capacity (Vacuumed, USA) filled with atmospheric air any time subjects inhaled for last time prior to each $\mathrm{BH}_{\mathrm{FI}}$. Subjects were verbally informed at $60 \mathrm{sec}, 30 \mathrm{sec}, 15 \mathrm{sec}$ prior to each $\mathrm{BH}_{\mathrm{FI}}$ and at $10 \mathrm{sec}$ a final sound signal reminded subjects to raise their head and the pillow was removed. At that point in time subjects voluntarily fully exhaled to their residual volume, inhaled $85 \%$ of their force vital capacity, [while the mouthpiece with the bag was removed away by a researcher] and immediately immersed their face, including chin and forehead, in the water. Upon termination of each $\mathrm{BH}_{\mathrm{FI}}$ effort, subjects raised their head above the water surface, turned their head right and a standby researcher inserted the mouthpiece in a proper way into their mouth for exhalation while the cover pillow was repositioned for head resting. After that subjects' face was dried gently with a towel of micro fibers (Packtowl Personal, MSR, USA).

Two min after the fifth $\mathrm{BH}_{\mathrm{FI}}$ effort subjects performed a HCVR test. Taking into account the starting time of the HCVR in the $1^{\text {st }}$ visit, the starting time of the $2^{\text {nd }}$ laboratory visit was determined about $\sim 30$ min earlier so both HCVR tests were performed, at approximately the same time [18]. HCVR test in any condition was always conducted in a sitting position. The subjects initially breathed atmospheric air through a mouthpiece with two manual three way valves connected directly to automated open-circuit spirometer in order to record breath-by-breath $\dot{\mathrm{V}} \mathrm{E}$ and $\mathrm{P}_{\mathrm{ET}} \mathrm{CO}_{2}$ as previously described. After that, subjects fully exhaled to their residual volume and stopcock was switched [manually by the researcher] to let the subject rebreath via an elastic vinyl bag also connected directly to automated open-circuit spirometer. The bag was filled with hyperoxic gas $\left[\sim 94 \% \mathrm{O}_{2}\right]$ enriched with $\mathrm{CO}_{2}[\sim 6 \%]$ where partial pressure of $\mathrm{CO}_{2}\left(\mathrm{PCO}_{2}\right)$ was approximately two times fold the estimated arteriovenous difference above rest $\mathrm{P}_{\mathrm{ET}} \mathrm{CO}_{2}$, ( $\sim 50 \mathrm{mmHg}$ for technical reasons). Subjects rebreathed from the bag until $\mathrm{P}_{\mathrm{ET}} \mathrm{CO}_{2}$ reached $65 \mathrm{mmHg}$ for 4 min minimum to $6 \mathrm{~min}$ maximum. Analysis of the breath-by-breath respiratory data revealed the $\mathrm{CO}_{2}$ sensitivity as expressed by the slope of $\alpha=\dot{\mathrm{V}} \mathrm{E} / \mathrm{PETCO}_{2}$ ), (for details and validity of HCVR test see original papers $[1,19])$. From the next day and for the next two weeks the NBH group only, was subjected to daily, under supervision, apnoea training in a series of five maximal repeated apnoea efforts separated by 2 min intervals. Subjects were sitting at rest or in supine position in a dry environment. In a third laboratory visit (POST condition) two weeks afterwards the NBH group repeated the experimental protocol of the second visit at the same time.

During second and third condition diaphragmatic activity was recorded continuously by skin-surface electromyography. Disposable, self-sticking silver/silver chloride electrodes $[+,-]$ Kendall Meditrace ${ }^{\mathbb{Q}}$ (Covidien, USA), were attached on the skin at the diaphragm level and were connected on line with a differential amplifier (EMG100 Biopack Systems Inc., USA), an analog to digital transducer (UIM100C Biopack Systems Inc., USA), the MP100A-CE system (Biopack Systems Inc., USA) and finally with a laptop (HP-Pavilion DV4000, Hewlett-Packard Company, USA). The MP100A was accompanied by AcqKnowledge 3.7.3 software (Biopack Systems Inc., USA), which was used during experimental procedure and for later electromyogram analysis. The electrical analog signal produced by the diaphragm was recorded at $1000 \mathrm{kHz}$. Later on a $20-250 \mathrm{kHz}$ band pass digital filter was applied followed by signal rectification [20], mean value smoothing (smoothing factor: 20) [21] and finally integration (for more details see AcqKnowledge $^{\circledR}$ III [22]). During $\mathrm{BH}_{\mathrm{FI}}$ efforts a recorded steep increase of diaphragmatic activity was considered as the landmark of involuntary ventilatory activity, and hence as the end of easy going phase (EPh). Steep rise was determined as an increase of two standard deviations above the mean value of the rest condition in diaphragmatic electromyogram. For breath hold time recording two hand 
stopwatches (Casio HS30W, Japan) were used. Arterialized capillary blood gases (IL 1640, Instrumentation Laboratory, Spain) measurements from the left middle finger were also conducted during rest and immediately after the end of each fifth $\mathrm{BH}_{\mathrm{FI}}$ effort. Hemodynamics and arterial hemoglobin oxygen saturation were also continuously recorded with a cuff attached on the right middle finger (Finommeter, Finapres Medical Systems BV, The Netherlands) and a pulse oximeter (NELLCOR Symphony N-3000, USA) on the left index finger respectively, mainly for safety reasons. Prior to each experimental condition, all instruments were calibrated according to manufacturers' guidelines. All subjects visited the laboratory having performed no strenuous activity in the preceding three days and kept similar food habits throughout the experimental design [23, 24]. All experiments were conducted at similar ambient air temperature $\left(24-25^{\circ} \mathrm{C}\right)$ and relative humidity $(50 \pm 5 \%)$.

The protocol of the study was complied with the Declaration of Helsinki [25] and was approved by local ethical committee. All participants were informed with details in a written way about any potential risk involved, as well as the experimental procedure and methods.

\section{Statistical Analysis}

All variables are presented as means \pm SE. Shapiro-Wilks tests and Q - Q graphs of dependent variables indicated that data were normally distributed $(\mathrm{P}>0.05)$. ANOVA with two factors was used for analyzing average BHT, HCVR and respiratory data. One factor entailed two independent levels (experienced or novice group; different subjects in each group, EBH vs $\mathrm{NBH}$ ). The other factor entailed three dependent levels (repeated measures of the same subjects) during visit 1,2 , and 3 . Data collected during repeated breath hold efforts were analyzed with a $2(\mathrm{EBH}, \mathrm{NBH}) \times 2$ (Pre, Post training) x 5 (BH efforts) ANOVA design with repeated measures on second and third level; Bonferroni post hoc tests were used whenever significant main effect or interaction was found. Independed $t$ test was used for comparisons in one variable between groups and correlation coefficient ( $\mathrm{r}$ ) between two variables. A $\mathrm{P} \leq 0.05$ was considered to indicate a statistically significant difference.

\section{RESULTS}

Physical traits of subjects are shown on Table 1. Subjects' BHT range was 274.0-400.0, 57.1-166.5 and 90.1$260.9 \mathrm{sec}$ in the EBH, PRE, and NBH, PRE, and POST conditions, respectively. The highest value of BHT was observed within the space among the third and the fifth $\mathrm{BH}_{\mathrm{FI}}$ effort ( $\mathrm{P} \leq 0.05$, Fig. 1). On the average BHT per each repeated $\mathrm{BH}_{\mathrm{FI}}$ effort was significantly higher in the $\mathrm{EBH}$, PRE than the NBH PRE, and POST conditions, $(\mathrm{P} \leq 0.05$, Fig. 1). The cumulative $\mathrm{BHT}$ of five $\mathrm{BH}_{\mathrm{FI}}$ efforts in the EBH, PRE condition $(1419.8 \pm 55.2 \mathrm{sec})$ was significantly higher compared to PRE $(539.0 \pm 50.0 \mathrm{sec})$, and POST $(720.9 \pm 74.9 \mathrm{sec})$ condition in NBH subjects, $(\mathrm{P} \leq 0.05)$. The cumulative BHT of NBH group in POST was also significantly improved by $33.8 \%$ compared to PRE condition, $(\mathrm{P} \leq 0.05)$.

The EBH had also significantly higher average values of $\mathrm{EPh}$ for each repeated $\mathrm{BH}_{\mathrm{FI}}$ effort than the $\mathrm{NBH}$, in PRE and POST conditions and the higher values were exhibited among the second and the fifth $\mathrm{BH}_{\mathrm{FI}},(\mathrm{P} \leq 0.05$, Fig. 2). The cumulative $\mathrm{EPh}$ of the five $\mathrm{BH}_{\mathrm{FI}}$ efforts was significantly higher in EBH, PRE condition $(669.3 \pm 59.4 \mathrm{sec})$ compared to NBH for both PRE (314.0 $\pm 37.4 \mathrm{sec})$ and POST (361.7 \pm $41.9 \mathrm{sec})$ conditions, $(\mathrm{P} \leq 0.05)$, while POST, did not differ significantly from PRE values, in NBH group.

On the average, recovery values in the 2 min relaxing periods of $\mathrm{P}_{\mathrm{ET}} \mathrm{CO}_{2}$ after repeated $\mathrm{BH}_{\mathrm{FI}}$, were significantly lower in $\mathrm{EBH}$, than in NBH group in both conditions, $\left(\mathrm{P} \leq 0.05\right.$, Fig. 3). Average $\dot{\mathrm{V}}_{E}$ values in the 2 min relaxing periods between repeated $\mathrm{BH}_{\mathrm{FI}}$ efforts were similar in all conditions and groups where subjects were instructed to avoid hyperventilation.

Hypercapnic response, determined by the slope $\alpha$ ( $\dot{\mathrm{V}} \mathrm{E} / \mathrm{PETCO}_{2}$ relationship), was higher $(1.8 \pm 0.2 \mathrm{l} / \mathrm{min} / \mathrm{mmHg})$ in BM than in PRE condition $(1.4 \pm 0.21 / \mathrm{min} / \mathrm{mmHg})$ in EBH group ( $\mathrm{P} \leq 0.05$, Fig. 4) since it decreased by $20.8 \%$ on the average in the latter condition. In the NBH group, the slope of $\alpha$ was $2.4 \pm 0.3,2.9 \pm 0.4$, and $2.8 \pm 0.5$ $1 / \mathrm{min} / \mathrm{mmHg}$ in BM, PRE and POST conditions, respectively (Fig. 4). The mean value of the slope $\alpha$, in BM condition of EBH group corresponded to $\sim 75 \%$ of the value of NBH group but there was no statistically significant difference (Fig. 4). However, the slope $\alpha$ of EBH group in PRE condition compared to PRE and POST conditions of the NBH group was significantly lower and accounted for $\sim 50 \%$ of the respective values. A high correlation $\mathrm{r}=0.61$, $(\mathrm{P} \leq$ 0.05 ) was also found between the slope $\alpha$ and the cumulative $\mathrm{EPh}$ of five repeated apnoeas of the EBH subjects in PRE condition; nothing similar was observed in NBH subjects (Table 2). Also, the partial pressure of arterialized $\mathrm{CO}_{2}$ after the five repetitive apnoeas was significantly higher than rest in both groups (Fig. 5).

Table 1. Physical characteristics of elite breath hold divers (EBH) and novice in apnoea subjects (NBH).

\begin{tabular}{|c|c|c|c|c|c|c|}
\hline \multicolumn{7}{|c|}{ EBH } \\
\hline & $\begin{array}{c}\text { Height } \\
(\mathbf{c m})\end{array}$ & $\begin{array}{c}\text { Mass } \\
(\mathbf{k g})\end{array}$ & $\begin{array}{c}\mathbf{A g e}^{*} \\
(\mathbf{y e a r s})\end{array}$ & $\begin{array}{c}\mathbf{F M}^{*} \\
\mathbf{( \% )}\end{array}$ & $\begin{array}{c}\mathbf{F V C}^{*} \\
(\mathbf{l})\end{array}$ & $\begin{array}{c}\text { VO } 2 \text { max } \\
(\mathbf{m l} / \mathbf{k g} / \mathbf{m i n})\end{array}$ \\
\hline \hline M & 182.5 & 84.4 & 28.1 & 16.6 & 6.9 & 42.8 \\
\hline SEM & 1.7 & 3.2 & 1.9 & 1.8 & 0.3 & 1.5 \\
\hline \multicolumn{7}{|c|}{$\mathbf{\text { NBH }}$} \\
\hline \hline M & 180.2 & 82.2 & 20.9 & 11.5 & 6.1 & 42.2 \\
\hline SEM & 2.1 & 3.6 & 0.5 & 1.3 & 0.1 & 1.9 \\
\hline
\end{tabular}

FM: fatty mass, FVC: force vital capacity, $\dot{\mathrm{V}}_{2 \max }$ : maximum oxygen uptake. ${ }^{*}$ Significant difference between groups, $(\mathrm{P} \leq 0.05)$.

\section{DISCUSSION}

Alteration of regular hypercapnic sensitivity, usually encountered in diving, mining and pulmonary diseases, is associated with fitness and wellness of respiration. Whereas 


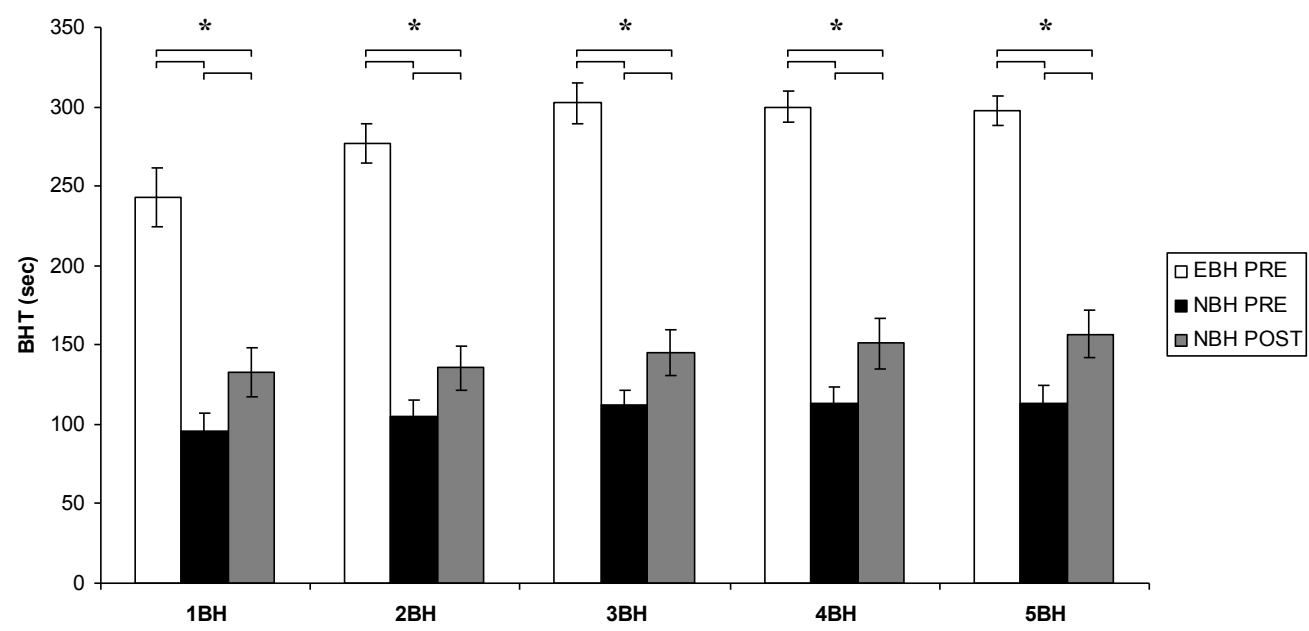

Fig. (1). Mean values $( \pm \mathrm{SE})$ of maximum breath hold time (BHT) in five repeated apnoeas $(\mathrm{BH})$ in pre training condition $(\mathrm{PRE})$ and after apnoea training intervention (POST), in elite breath hold divers (EBH) and novice in apnoea subjects (NBH). [The * indicates significant difference between conditions, $(\mathrm{P} \leq 0.05)]$.

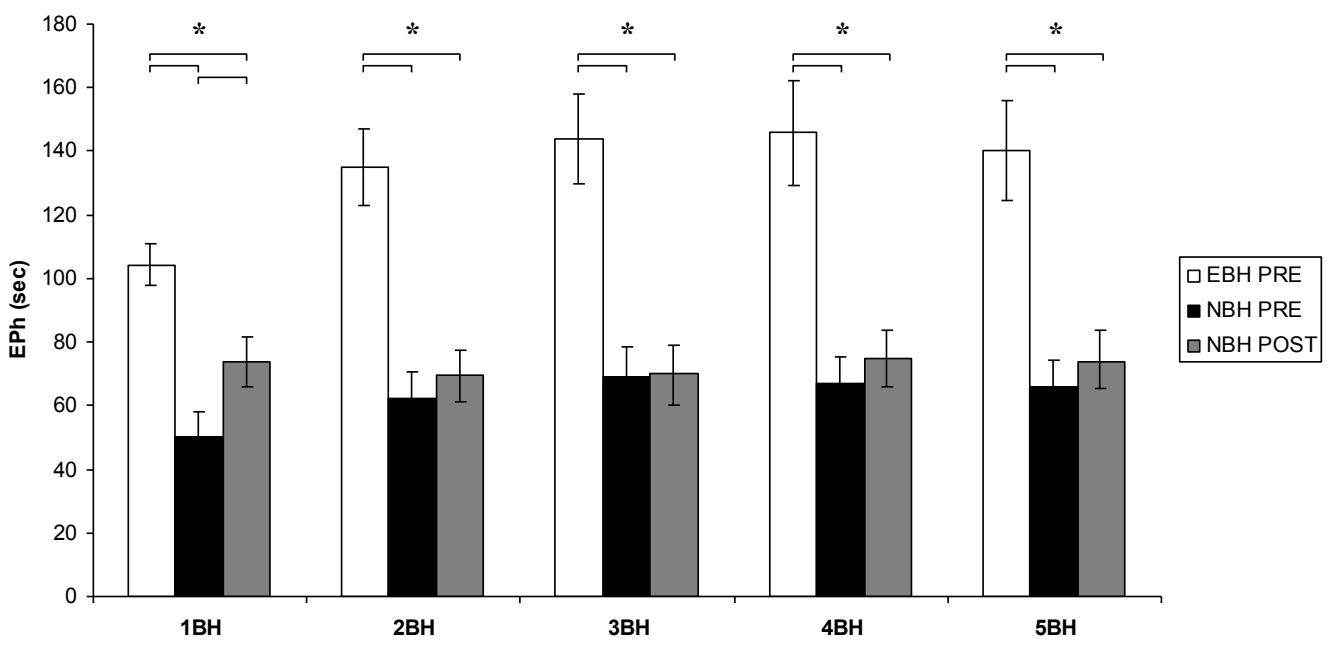

Fig. (2). Mean values $( \pm \mathrm{SE})$ of easy going phase $(\mathrm{EPh})$ in five repeated apnoeas $(\mathrm{BH})$ in pre training condition $(\mathrm{PRE})$ and after apnoea training intervention (POST), in elite breath hold divers (EBH) and novice in apnoea subjects $(\mathrm{NBH})$. [The * indicates significant difference between conditions, $(\mathrm{P} \leq 0.05)]$.

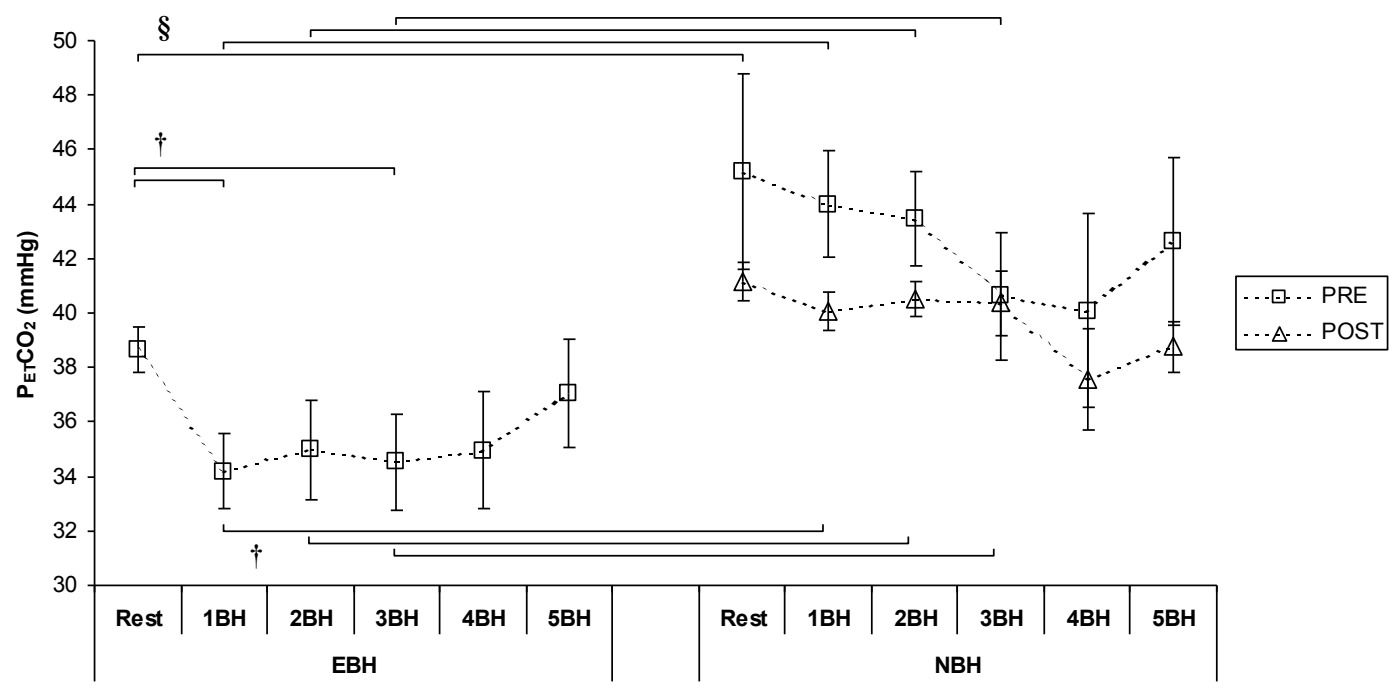

Fig. (3). Mean values $( \pm \mathrm{SE})$ of partial pressure of end-tidal carbon dioxide $\left(\mathrm{P}_{\mathrm{ET}} \mathrm{CO}_{2}\right)$ at rest (Rest) and after five repetitive apnoeas $(\mathrm{BH})$ in pre training condition (PRE) or after the apnoea training intervention (POST) in the elite breath hold divers (EBH) and novice in apnoea subjects $(\mathrm{NBH})$. [The $\dagger, \S$ indicate significant differences within or between BH efforts in PRE conditions, and between groups in PREPOST contrast, respectively, $(\mathrm{P} \leq 0.05)]$. 


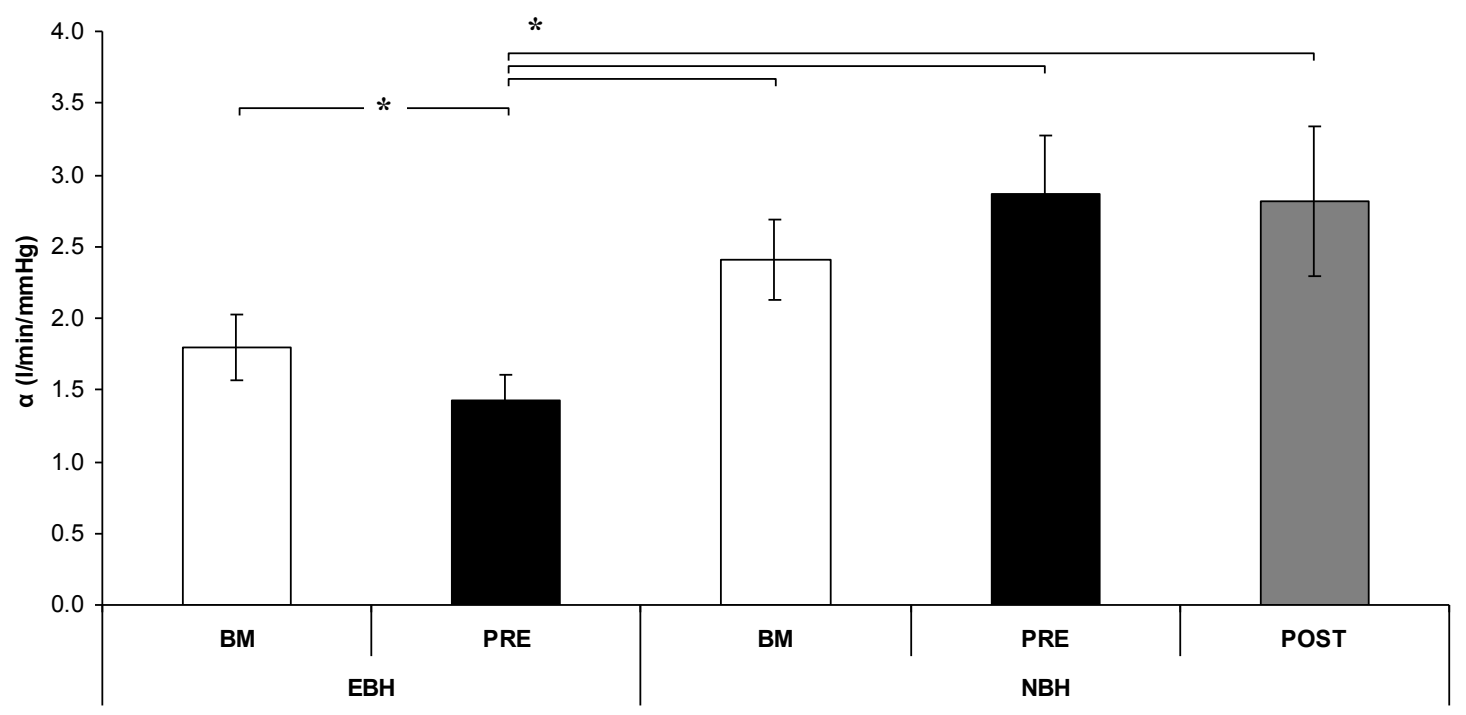

Fig. (4). Mean values ( $\pm \mathrm{SE}$ ) of measurement of $\mathrm{CO}_{2}$ sensitivity as expressed by the slope of $\alpha=\dot{\mathrm{V}}_{\mathrm{E}} / \mathrm{PeTCO}_{2}$, in base line condition (BM), in the pre training (PRE) and after apnoea training intervention (POST), in elite breath hold divers (EBH) and novice in apnoea subjects (NBH). [The * indicates significant difference between conditions, $(\mathrm{P} \leq 0.05)]$.

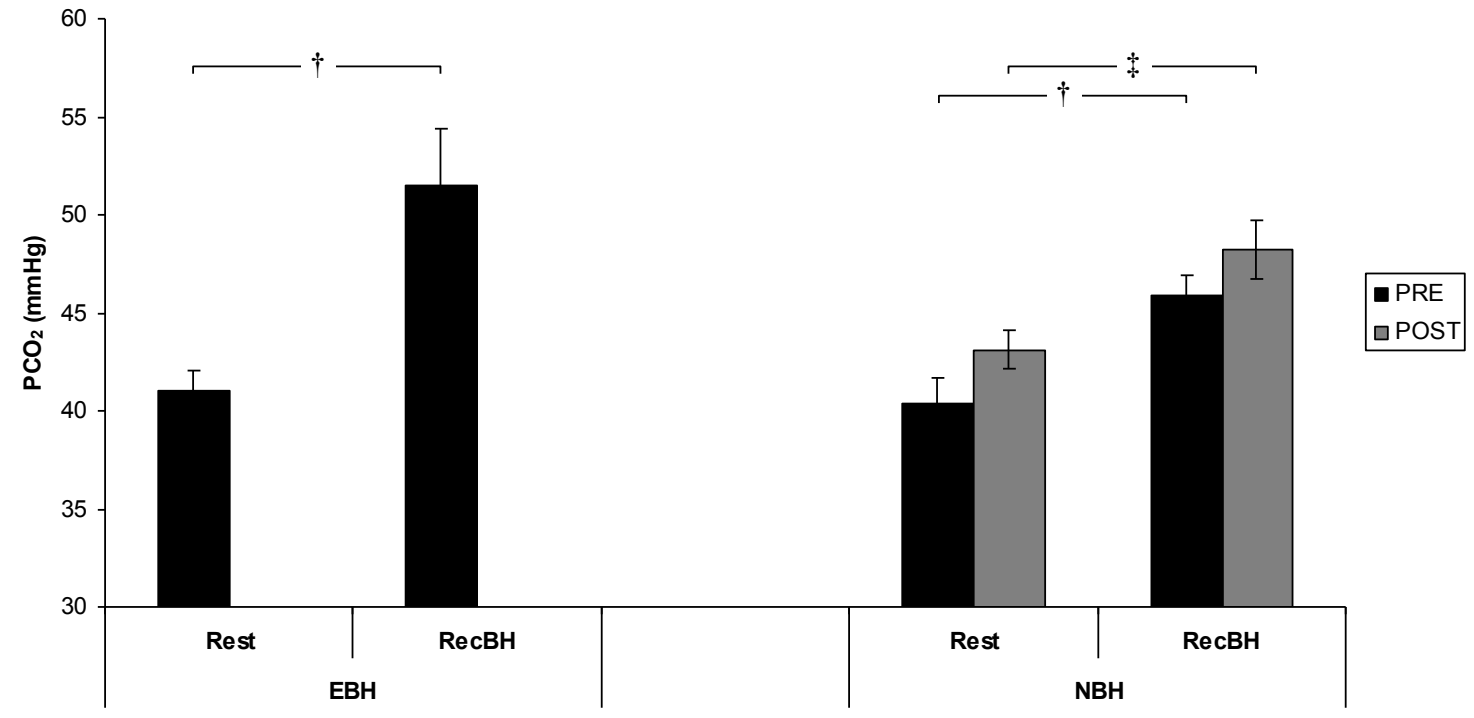

Fig. (5). Mean values $( \pm \mathrm{SE})$ of measurement of partial pressure of arterialized $\mathrm{CO}_{2}\left(\mathrm{PCO}_{2}\right)$ at rest (Rest), after the five repetitive apnoeas $(\mathrm{recBH})$ in the pre training condition (PRE) and after the apnoea training intervention (POST) in the elite breath hold divers (EBH) and novice in apnoea subjects $(\mathrm{NBH})$. [The $\dagger$, $\$$, indicate significant differences between the BH efforts in PRE and POST conditions, respectively, $(\mathrm{P} \leq 0.05)]$.

Table 2. Correlation ( $r$ ) of the cumulative easy going phase of five repeated apnoeas in the pre training condition (PRE) and after apnoea training intervention (POST), in elite breath hold divers (EBH) and novice in apnoea subjects (NBH) with the slope $\alpha\left(\dot{\mathbf{V}}_{\mathrm{E}} / \mathbf{P}_{\mathrm{ET}} \mathrm{CO}_{2}\right)$, as expressed by measurement of the $\mathrm{CO}_{2}$ sensitivity, two minutes after the end of the five repeated apnoeas efforts.

\begin{tabular}{|c|c|}
\hline & $\mathbf{r}\left(\mathbf{r}^{2}\right)$ \\
\hline \hline EBH (PRE) & $0.61^{*}(0.37)$ \\
\hline NBH (PRE) & $-0.06(0.00)$ \\
\hline NBH (POST) & $-0.27(0.07)$ \\
\hline *Significant correlation among variables, $(\mathrm{P} \leq 0.05)$. \\
\hline
\end{tabular}

this fact is undoubtedly accepted, the process of developing hypercapnic dullness is vague and controversial. The present study tested whether hypercapnic ventilatory de-sensitization occurs after five intermittent apnoea efforts and in a mid term after two weeks of daily apnoea training sessions in healthy, experienced and novice divers. It was initially hypothesized that low hypercapnic ventilatory sensitivity is a long - drawn process, which befits only experienced skin divers and that short term training would not be effective in improving it. It was found that indeed experienced skin divers tend to have low carbon dioxide sensitivity, which is further enhanced after repeating five apnoea efforts. On the other hand, carbon dioxide sensitivity in novice apnoeists did not decrease after performing five intermittent breath hold efforts either in an acute way or after two weeks of apnoea training. 
The experimental protocol of five repeated static $\mathrm{BH}_{\mathrm{FI}}$ with a two minute interval in between efforts has been widely used $[8,10,26]$. Subjects in both EBH, PRE and $\mathrm{NBH}, \mathrm{POST}$ conditions, succeeded significant elongation of BHT which gradually peaked in the last two efforts, like a number of other studies with similar protocols have solidly confirmed [10-15, 27-29]. As it was expected [30] EBH individuals accomplished, significantly higher values of BHT per each breath hold effort and in total compared to NBH subjects.

The great variance of BHT among $\mathrm{EBH}$ and $\mathrm{NBH}$ individuals mostly depends on the interaction of chemosensitivity, and psychological factors [31-33]. The onset of diaphragmatic involuntary ventilatory activity separates breath hold in two phases, the easy going and struggle phase $(\mathrm{SPh})[6,34,35]$. In $\mathrm{EPh}$, diaphragmatic activity is too mild whereas in SPh, diaphragmatic activity is intense as the effort for preserving apnoea increases dramatically $[6,34,35]$. The duration of $\mathrm{EPh}$ is determined by chemical stimuli such as the increased arterial partial pressure of carbon dioxide and the decreased arterial partial pressure of oxygen $[6,31,33,34]$, while $\mathrm{SPh}$ duration is determined by non chemical stimuli possibly such as the subjects' psychological strength to overcome the central command for ventilation and the unpleasant feeling of asphyxiation [36-38].

With regard to chemical stimuli, EBH and NBH subjects exhibited similar partial pressure of arterialized, and endtidal carbon dioxide values, at rest. Likewise, no difference recorded in HCVR tested before experimental evaluation, although there was a tendency $(\mathrm{P}=0.11)$ in the slope $\alpha$ to be lower in EBH than NBH. Hypercapnic de-sensitization became apparent in EBH subjects when HCVR was tested after a manoeuvre of performing five intermittent efforts of apnoea, a finding in accordance with the literature [7, 19, 3942]. On the contrary, the same manoeuvre rather worsens hypercapnic response in NBH. Comparing EBH and NBH, the lower chemosensitivity in EBH was accompanied by a lower end tidal carbon dioxide partial pressure measured in the mid point of the 2 min break between repeated apnoeas. This association between $\mathrm{CO}_{2}$ sensitivity and $\mathrm{P}_{\mathrm{ET}} \mathrm{CO}_{2}$ is enhanced after two weeks of apnoea training (see Figs. 3, 4). It is noteworthy, that arterialized carbon dioxide, measured right away the fifth apnoea effort, was elevated in both EBH and NBH groups, a finding previously shown [8]. These findings suggest that there is a sequence of physiological events underlying the development of carbon dioxide desensitization. It appears that high values of arterialized carbon dioxide constitute the stimuli in all cases. Low values of alveolar $\mathrm{CO}_{2}$, as seen in $\mathrm{EBH}$, constitutes a counter-acting stimulus to high arterial $\mathrm{CO}_{2}$ and probably contributes to the exhibited low chemosensitivity. The exact mechanism of improving arterial to alveolar gradient in order to eliminate high amounts of $\mathrm{CO}_{2}$ effectively at the background of low chemosensitivity, as it was found in EBH, is unknown, but it could be speculated that is caused by long drawn changes, which might improve lung diffusion capacity or ventilation perfusion ratio. In $\mathrm{NBH}$ such scheme is sketchy; it may gradually develop, however, as seen from the progressive drop of $\mathrm{P}_{\mathrm{ET}} \mathrm{CO}_{2}$ after two weeks of apnoea training.
The time period needed someone to develop low $\mathrm{CO}_{2}$ sensitivity is not known. It was only shown that after three months' absence from diving training chemosensitivity of instructors working at a submarine escape training tank increased [4]. Information entailing long term apnoea training on chemosensitivity is entirely lacking.

Chronic retention of $\mathrm{CO}_{2}$ in SCUBA divers [2, 40, 43-45] as well as in free divers [3-5] has been correctly associated with diminution of hypercapnic ventilatory response. However, the present results of novice apnoeists indicated that this is not always the case, as someone would assume based on Bakovic et al. (2006) data. In other words, in inexperienced subjects any temporary retention of $\mathrm{CO}_{2}$ succeeded five repeated $\mathrm{BH}_{\mathrm{FI}}$ does not have as a result a short-term decreased HCVR in order to delay the chemical stimulus to cease breath and in extension to increase BHT [7].

Another original finding of the present study was that HCVR was highly correlated with the cumulative easy going phase of five repeated apnoeas in EBH but not in NBH. Therefore, we could allege that BHT improvement in EBH group is attributed to changes in chemosensitivity whereas the respective improvement of BHT in NBH group is probably caused by improvement of tolerance of unpleasant stimuli. However, except the contribution of chemosensitivity and mettle in BHT elongation, after repeated $\mathrm{BH}_{\mathrm{FI}}$, unintentional hyperventilation between $\mathrm{BH}$ efforts, apnoeogenic chemodynamic adaptations and hematological changes due to active contraction of spleen, should also contribute in increase of BHT [7, 10, 11, 14, 29, $46,47]$. None of the above factors were measured, in the present study, except that no hyperventilation in between the $\mathrm{BH}_{\mathrm{FI}}$ was allowed.

For the first time the present study proved that dullness of hypercapnic ventilatory response after 5 repeated breath hold efforts is an exclusive trait, highly correlated with the cumulative easy going phase only in elite and not in novice skin divers. Furthermore, the increase of maximum breath hold time in novice subjects throughout five repeated apnoea efforts is rather attributed to elongation of struggle phase and to enhanced stamina for unpleasant stimuli. Hypercapnic desensitization is considered to serve energy parsimony by unloading respiratory hyperactivity. The principles of its development and the level of its tolerance have paramount importance in the treatment of respiratory diseases and the enhancement of apnoea performance. The minimum time period needed for someone to develop low hypercapnic ventilatory response remains unknown and further research is needed.

\section{CONFLICT OF INTEREST}

The authors confirm that this article content has no conflict of interest.

\section{ACKNOWLEDGEMENTS}

We greatly appreciate the technical assistance of Konstantinos I. Pavlakis.

\section{REFERENCES}

[1] Read DJ. A clinical method for assessing the ventilatory response to carbon dioxide. Australas Ann Med 1967; 16: 20-32. 
[2] Florio JT, Morrison JB, Butt WS. Breathing pattern and ventilatory response to carbon dioxide in divers. J Appl Physiol 1979; 46: 1076-80.

[3] Hong SK, Rahn H, Kang DH, Song SH, Kang BS. Diving pattern, lung volumes, and alveolar gas of the Korean diving woman (ama). J Appl Physiol 1963; 18: 457-65.

[4] Schaefer KE. Adaptation to breath hold diving. In: Rahn $\mathrm{H}$, Yokoama T, Eds. Physiology of breath hold diving and the ama of Japan. Washington, DC: National Academy of Sciences, National Research Counsil, Publication 1341; 1965: 237-52.

[5] Song SH, Kang DH, Kang BS, Hong SK. Lung volumes and ventilatoryresponses to high $\mathrm{CO}_{2}$ and low $\mathrm{O}_{2}$ in the ama. J Appl Physiol 1963; 18: 466-70.

[6] Lin YC, Lally DA, Moore TO, Hong SK. Physiological and conventional breath-hold breaking points. J Appl Physiol 1974; 37 : 291-6.

[7] Chang LP, Lundgren CE. Maximal breath-holding time and immediate tissue $\mathrm{CO}_{2}$ storage capacity during head-out immersion in humans. Eur J Appl Physiol Occup Physiol 1996; 73: 210-18.

[8] Bakovic D, Eterovic D, Valic Z, et al. Increased pulmonary vascular resistance and reduced stroke volume in association with $\mathrm{CO}_{2}$ retention and inferior vena cava dilatation. J Appl Physiol 2006; 101: 866-72.

[9] Ferretti G, Costa M. Diversity in and adaptation to breath-hold diving in humans. Comp Biochem Physiol A Mol Integr Physiol 2003; 136: 205-13.

[10] Schagatay E, van Kampen M, Andersson J. Effects of repeated apneas on apneic time and diving response in non-divers. Undersea Hyperb Med 1999; 26: 143-9.

[11] Schagatay E, Andersson JP, Hallen M, Palsson B. Selected contribution: role of spleen emptying in prolonging apneas in humans. J Appl Physiol 2001; 90: 1623-9.

[12] Schagatay E, Haughey H, Reimers J. Speed of spleen volume changes evoked by serial apneas. Eur J Appl Physiol 2005; 93: 447-52.

[13] Bakovic D, Valic, Z, Eterovic D, et al. Spleen volume and blood flow response to repeated breath-hold apneas. J Appl Physiol 2003; 95: 1460-66.

[14] Bakovic D, Eterovic D, Saratlija-Novakovic Z, et al. Effect of human splenic contraction on variation in circulating blood cell counts. Clin Exp Pharmacol Physiol 2005; 32: 944-51.

[15] Richardson M, de Bruijn R, Holmberg HC, Bjorklund G, Haughey $\mathrm{H}$, Schagatay E. Increase of hemoglobin concentration after maximal apneas in divers, skiers, and untrained humans. Can J Appl Physiol 2005; 30: 276-81.

[16] Andersson J, Schagatay E. Repeated apneas do not affect the hypercapnic ventilatory response in the short term. Eur J Appl Physiol 2009; 105: 569-74.

[17] Bartlett D Jr. Effects of Valsalva and Mueller maneuvers on breathholding time. J Appl Physiol 1977; 42: 717-21.

[18] Bosco G, Ionadi A, Data PG, Mortola JP. Voluntary breath-holding in the morning and in the evening. Clin Sci (Lond) 2004; 106: 34752 .

[19] Read DJ, Leigh J. Blood-brain tissue $\mathrm{PCO}_{2}$ relationships and ventilation during rebreathing. J Appl Physiol 1967; 23: 53-70.

[20] Petrofsky JS. Frequency and amplitude analysis of the EMG during exercise on the bicycle ergometer. Eur J Appl Physiol Occup Physiol 1979; 41: 1-15.

[21] Kamen G, Caldwell GE. Physiology and interpretation of the electromyogram. J Clin Neurophysiol 1996; 13: 366-84.

[22] Biopack Systems I. AcqKnowledge ${ }^{\circledR}$ III for the MP100WS. Operating Manual 3.2 ed. USA: Biopack Systems, Inc. 1995.

[23] Lindholm P, Conniff M, Gennser M, Pendergast D, Lundgren C. Effects of fasting and carbohydrate consumption on voluntary resting apnea duration. Eur J Appl Physiol 2007; 100: 417-25.
[24] Tsintzas OK, Williams C, Boobis L, Greenhaff P. Carbohydrate ingestion and glycogen utilization in different muscle fibre types in man. J Physiol 1995; 489(Pt1): 243-50.

[25] World Medical Association. World Medical Association (WMA) Declaration of Helsinki. In: Ethical Principles for Medical Research Involving Human Subjects. Seoul: World Medical Association 2008; pp. 1-5.

[26] Schagatay E, Andersson J. The respiratory, circulatory and hematological effects of repeated apneas in humans. Undersea Hyperb Med 1999; 26: 29-30.

[27] Prommer N, Ehrmann U, Schmidt W, Steinacker JM, Radermacher P, Muth CM. Total haemoglobin mass and spleen contraction: a study on competitive apnea divers, non-diving athletes and untrained control subjects. Eur J Appl Physiol 2007; 101: 753-9.

[28] Schagatay E, van Kampen M, Emanuelsson S, Holm B. Effects of physical and apnea training on apneic time and the diving response in humans. Eur J Appl Physiol 2000; 82: 161-9.

[29] Heath JR, Irwin CJ. An increase in breath-hold time appearing after breath-holding. Respir Physiol 1968; 4: 73-7.

[30] AIDA International. World Records; 2010. Internet [On-line (5/5/2024)]. Available from: http://www.aidainternational.org/com petitive/worlds-records

[31] Godfrey S, Campbell EJ. The control of breath holding. Respir Physiol 1968; 5: 385-400.

[32] Godfrey S, Campbell EJ. Mechanical and chemical control of breath holding. Q J Exp Physiol Cogn Med Sci 1969; 54: 117-28.

[33] Courteix D, Bedu M, Fellmann N, Heraud MC, Coudert J. Chemical and nonchemical stimuli during breath holding in divers are not independent. J Appl Physiol 1993; 75: 2022-7.

[34] Agostoni E. Diaphragm activity during breath holding: factors related to its onset. J Appl Physiol 1963; 18: 30-36.

[35] Dejours P. Control of respiration in muscular exercise. In: Fenn WO, Rahn H, Eds. Handbook of physiology, sect 3 . The respiratory system. Washington: American Physiological Society 1964: 63148 .

[36] Schneider EC. Observation on holding the breath. Am J Physiol 1930; 94: 464-70.

[37] White PD. Observations on some tests of physical fitness. Am J Med Sci 1920; 159: 866-74

[38] Rigg JR, Rebuck AS, Campbell EJ. A study of factors influencing relief of discomfort in breath-holding in normal subjects. Clin Sci Mol Med 1974; 47: 193-9.

[39] Hirshman CA, McCullough RE, Weil JV. Normal values for hypoxic and hypercapnic ventilaroty drives in man. J Appl Physiol 1975; 38: 1095-8.

[40] Sherman D, Eilender E, Shefer A, Kerem D. Ventilatory and occlusion-pressure responses to hypercapnia in divers and nondivers. Undersea Biomed Res 1980; 7: 61-74.

[41] Bjurstrom RL, Schoene RB. Control of ventilation in elite synchronized swimmers. J Appl Physiol, 1987; 63: 1019-24.

[42] Masuda Y, Yoshida A, Hayashi F, Sasaki K, Honda Y. The ventilatory responses to hypoxia and hypercapnia in the Ama. Jpn J Physiol 1981; 31: 187-97.

[43] Kerem D, Melamed Y, Moran A. Alveolar $\mathrm{PCO}_{2}$ during rest and exercise in divers and non-divers breathing $\mathrm{O}_{2}$ at 1 ATA. Undersea Biomed Res 1980; 7: 17-26.

[44] Morrison JB, Florio JT, Butt WS. Effects of $\mathrm{CO}_{2}$ insensitivity and respiratory pattern on respiration in divers. Undersea Biomed Res 1981; 8: 209-17.

[45] Lally DA, Zechman FW, Tracy RA. Ventilatory responses to exercise in divers and non-divers. Respir Physiol 1974; 20: 117-29.

[46] Hentsch U, Ulmer HV. Trainability of underwater breath-holding time. Int J Sports Med 1984; 5: 343-7.

[47] Vasar E, Kingisepp PH. Physiological characteristics of repeated breath holding. Adv Physiol Sci 1980; 10: 639-46. 